\title{
Relações entre afetos positivos e negativos e os cinco fatores de personalidade
}

Ana Paula Porto Noronha. Universidade São Francisco. Denise da Fonseca Martins. Universidade São Francisco. Roberta Ramazotti Ferraz Campos. Universidade São Francisco. Camélia Santina Murgo Mansão. Universidade do Oeste Paulista.

\section{Resumo}

A presente pesquisa tem como objetivo verificar a existência de associações entre afetos positivos e negativos e os fatores de personalidade, sob a perspectiva do modelo dos Cinco Grandes Fatores. Colaboraram para o desenvolvimento do estudo 529 estudantes do Ensino Médio de escolas públicas e particulares com idades entre 14 a 27 anos $(M=16,0 ; D P=1,48)$. No que se refere ao sexo 223 (42,2\%) eram homens e 306 (57,8\%), mulheres. Foram aplicadas a Escala de Afetos Zanon (EAZ) e a Bateria Fatorial de Personalidade (BFP). Dentre os resultados, a relação entre o fator neuroticismo e os afetos negativos gerou o coeficiente mais forte $(r=0,62)$. Em relação aos afetos positivos, a maior correlação se deu com o fator extroversão $(r=0,42)$. Verificou-se que neuroticismo é o melhor preditor dos afetos negativos, enquanto que extroversão dos afetos positivos. Os achados são discutidos à luz da literatura.

Palavras-chave: psicologia positiva; bem-estar subjetivo; traços de personalidade; afetos; ajustamento emocional.

\begin{abstract}
Relationship between positive and negative affects and the big five. The present research shows the correlations analyses result between positive and negative affects and the big five. Contributed to the development of study 529 students from public and private high schools, aged between 14 and 27 years $(M=16.0 ; S D=1.48)$. About the gender $223(42.2 \%)$ were males and 306 (57.8\%) were females. The instruments used were the 'Escala de Afetos Zanon (EAZ)' and the 'Bateria Fatorial de Personalidade (BFP)'. About the results, the relation between the neuroticism factor and the negative affect was the stronger coefficient $(r=0,62)$. The positive affect, showed a stronger correlation with the extroversion factor $(r=0,42)$. It was found that neuroticism is the best predictor of negative affect, while extroversion of positive affect. The findings are discussed in the literature context. Keywords: positive psychology; subjective well-being; personality traits; affects; emotional adjustment.
\end{abstract}

\section{Resumen}

Relaciones entre afectos positivos y negativos y los cinco factores de personalidad. La presente investigación presenta el resultado del análisis correlacional entre afectos positivos y negativos y los factores de personalidad, sobre la perspectiva del modelo de los Cinco Grandes Factores. Colaboraron para el desarrollo del estudio 529 estudiantes de Enseñanza Secundaria de escuelas públicas y privadas, con edades entre 14 y 27 años $(M=16,0 ; D T=1,48)$. En relación al sexo, $223(42,2 \%)$ eran hombres y 306 (57,8\%) mujeres. Fueron aplicadas la Escala de Afectos Zanon (EAZ) y la Batería Factorial de Personalidad (BFP). Entre los resultados, el coeficiente más fuerte fue entre el factor neuroticismo y los afectos negativos $(r=0,62)$. En relación a los afectos positivos, la mayor correlación se dio con el factor extraversión $(r=0,42)$. Se verificó que el neuroticismo es el que mejor predice los afectos negativos, mientras que la extraversión predice mejor los afectos positivos. Los resultados son discutidos con base en la literatura. Palabras clave: psicología positiva; bienestar subjetivo; rasgos de personalidad; afectos; ajuste emocional. 
Não são recentes as indagações do homem acerca do que torna sua vida feliz. A medida da felicidade parece estar diretamente associada à maneira como as pessoas avaliam cognitiva e afetivamente a própria vida (Seligman, 2011). A Psicologia Positiva tem como interesse o entendimento do que torna a vida gratificante, mais especialmente, do bem-estar subjetivo. Segundo Seligman (2004), a Psicologia Positiva deveria ser compreendida à luz de três pilares principais, quais sejam, as emoções positivas (como felicidade, esperança e alegria); as qualidades positivas (como caráter, forças e virtudes); e as instituições positivas (como locais de trabalho, escolas, famílias, hospitais, comunidades ou sociedades). Especialmente quanto às emoções positivas, elas têm sido compreendidas como uma versão mais específica do humor, e composta pelos afetos (Snyder \& Lopes, 2009), que serão objeto de investigação do presente estudo.

De acordo com Warner e Rasco (2014), o bem-estar subjetivo é composto por três fatores: satisfação com a vida (componente cognitivo), afeto positivo e afeto negativo (componentes afetivos). Inclui, portanto, as reações emocionais frente aos eventos do cotidiano e os julgamentos cognitivos de satisfação ou insatisfação com a vida, tal como pontuado por Diener, Lucas e Oishi (2002). O afeto é composto pelas respostas afetivas das pessoas e se apresenta em duas dimensões: positivo e negativo. $O$ positivo reflete o quanto uma pessoa sente-se entusiasmada, ativa e alerta, enquanto o negativo diz respeito à angústia, insatisfação, estados de humor aversivos como raiva, culpa, desgosto e medo (Watson, Clark, \& Tellegen, 1988).

Alcalá, Camacho, Giner, Giner e Ibañez (2006) afirmam que as dimensões dos afetos embora sejam duas, são relacionadas, porém independentes. Uma revisão sobre o construto foi elaborada por Segabinazi et. al (2012), na qual apontam que coube a Costa e McCrae (1980), os estudos iniciais com a PANAS (Watson et al., 1988), cujos resultados levaram à compreensão de que os afetos positivos e negativos seriam pólos opostos do construto, o que explicaria sua independência. Os afetos implicam, além dos mecanismos fisiológicos referenciados por Snyder e Lopez (2009), componentes cognitivos, expressões comportamentais e variáveis sociais e culturais. Ademais, níveis elevados de afetos positivos indicam a experimentação frequente de alegria, entusiasmo, confiança, e engajamento no cumprimento de tarefas, enquanto altos escores em afetos negativos revelam experiências repetidas de tristeza, desânimo e preocupação.
Diversas variáveis têm sido consideradas na tentativa de ampliação das explicações sobre o que determina prevalência de afetos positivos ou negativos. A maioria dessas variáveis tem caráter sociodemográfico, ou seja, investigações buscaram encontrar correlações entre afetos e gênero, idade, contexto cultural (Brajša-Žganec \& Kaliterna-Lipovčan, 2006; Diener \& Biswas-Diener, 2000; Inglehart, 2002), dentre outros. No entanto, os estudos também mostraram que os fatores demográficos não explicam variância do bem-estar subjetivo com tanto sucesso como esperado (Argyle, 1999; Inglehart, 2002; Inglehart \& Klingemann, 2000). Em contrapartida, traços de personalidade são apontados como responsáveis por um terço da variação do bem-estar subjetivo (Chan \& Joseph, 2000; Garcia \& Erlandsson, 2011; Lucas \& Diener, 2008). A este respeito, Wilson (1967) há quase quatro décadas afirmou que personalidade e características demográficas contribuem com a felicidade, de modo que jovens saudáveis, bem educados, extrovertidos e otimistas tendem a ser mais felizes.

Um modelo teórico de compreensão da personalidade que vem sendo relacionado ao bem-estar subjetivo é denominado Big Five ou Modelo dos Cinco Grandes Fatores (McCrae \& John, 1992; Nunes \& Hutz, 2002). A personalidade é apresentada por meio de cinco dimensões globais ou fatores, a saber, Neuroticismo, Extroversão, Abertura a Experiências, Socialização e Realização. Cada fator, por sua vez, compreende diversas facetas representativas de características específicas do fator correspondente.

No que se refere à relação entre personalidade e afetos, Albuquerque, Lima, Matos e Figueiredo (2013), pontuam que o estudo da personalidade é a maneira mais eficaz para a compreensão do bem-estar. Zanon e Hutz (2010) discorrem que existem interrelações entre os construtos, e que o modo como eles se configuram nas pessoas tende a manter-se durante a vida, o que acontece, segundo os autores, da mesma forma com os traços de personalidade e o bem-estar subjetivo. Isso posto, torna-se plausível pensar que os fatores do Big Five possam em alguma medida associarem-se com os afetos positivos e negativos, uma vez que estes são elementos constituintes do bem-estar subjetivo.

Corroborando tais afirmações, Anglim e Grant (2014) apontam evidências de que os fatores de personalidade Extroversão, Socialização e Neuroticismo predizem o bem-estar subjetivo, sendo que o fator Extroversão está fortemente correlacionado com o afeto positivo, e Neuroticismo tem sido associado com baixa satisfação de vida 
e afeto negativo. Os dados foram verificados por meio da pesquisa dos autores que contaram com a participação de 337 pessoas de duas universidades australianas, com idades entre 16 e 55 anos ( $M=21, D P=8,8)$, sendo $76 \%$ do sexo feminino. Foram utilizados os instrumentos IPIP, Escala de Bem-estar Psicológico, Escala de Satisfação com a Vida e a PANAS (Positive and Negative Affect Scale). Os resultados apontaram que Neuroticismo apresentou correlação com maior magnitude com afeto negativo $(r=0,76 ; p=0,05)$, indicando uma tendência a experimentar uma série de emoções negativas. Por outro lado, houve correlações entre Extroversão e afeto positivo ( $r=$ $0,56 ; p=0,05)$, o que pode estar relacionado à vivência de emoções positivas, englobando satisfação com a vida, relações positivas, crescimento pessoal e auto-aceitação. Outras pesquisas recentes com objetivos semelhantes serão apresentadas.

Em seu estudo Brajša-Žganec, Ivanović e Lipovčan (2011) examinaram a relação entre traços de personalidade, desejabilidade social e bem-estar subjetivo. Um total de 392 estudantes de uma universidade da Croácia (195 mulheres e 197 homens), com idades de 19 a 26 anos $(M=20,25, D P=1,46)$ responderam a Escala de Satisfação com a Vida, PANAS, Marlowe-Crowne Escala de Desejabilidade Social e medidas das cinco grandes dimensões da personalidade (IPIP50). Os resultados indicaram que traços de personalidade, especificamente Extroversão e Realização, representam fortes preditores de bem-estar subjetivo (satisfação com a vida, afetos positivos e negativos). As magnitudes mais fortes foram obtidas entre realização e bem-estar subjetivo, indicando que pessoas emocionalmente estáveis têm maior satisfação com a vida.

Albuquerque, Lima, Matos e Figueiredo (2012) realizaram uma pesquisa que contou com a participação de 398 professores portugueses do ensino fundamental e do ensino médio, sendo que 287 eram mulheres e 111 homens, com idade média de 41,09 anos ( $D P=7,71$ ). Para avaliar a personalidade, os autores utilizaram o Inventário de Personalidade NEO PI-R, e para a mensuração do bem-estar subjetivo a Escala de Satisfação com a Vida e a PANAS. Os resultados mostraram correlações estatisticamente significativas entre Neuroticismo e afeto negativo $(r=0,66 ; p<0,01)$, Extroversão e afeto positivo $(r=0,45$; $p<0,01$ ), bem como entre Realização e afeto positivo $(r=0,42)$. De acordo com os autores, Neuroticismo pode ser considerado como o melhor preditor dos afetos negativos, enquanto Extroversão está diretamente relacionada com os afetos positivos.
Nesse mesmo sentido, em contexto nacional, Zanon, Bastianello, Pacico e Hutz (2013a) investigaram as associações entre fatores de personalidade e afetos. Participaram 319 estudantes universitários com idades entre 17 e 37 anos ( $M=21,5, D P=4,9)$, sendo que $64 \%$ eram mulheres. Os mais fortes preditores de afetos negativos e positivos foram respectivamente Neuroticismo e Realização. Ao lado disso, Socialização apresentou pequena predição sobre os afetos positivos, mas nenhuma relação com negativos.

Também no Brasil, Nunes, Hutz e Giacomini (2009) investigaram as relações entre bem-estar subjetivo e os fatores Extroversão, Socialização e Neuroticismo em uma amostra composta por 357 estudantes universitários, de ambos os sexos. Os autores verificaram que, dentre as facetas de Extroversão, a que apresentou maior correlação com satisfação de vida e afeto positivo foi interação social, que descreve o quanto as pessoas buscam ativamente por situações que proporcionem contato social, bem como situações estimulantes. Não houve correlação significativa entre o fator e afeto negativo, embora tenha havido entre o último e Neuroticismo $(r=0,44 ; p<0,01)$. Depressão, faceta do fator Neuroticismo, apresentou a maior correlação negativa com satisfação de vida e maior correlação positiva com afeto negativo.

Para Mroczek e Kolarz (1998) as pessoas diferem em seus níveis de bem-estar subjetivo e afeto, dentre outras características, sendo que tais especificidades podem ser explicadas por variáveis contextuais, sociodemográficas, e mais representativamente, pelas características de personalidade. Nemanick e Munz (1997) problematizam que não há consenso na literatura entre a relação entre os fatores de personalidade e os afetos positivos e negativos. Em alguma medida a divergência pode ser mais bem explicada pelos dois estudos de meta-análise apresentados a seguir.

DeNever e Cooper (1998) realizaram uma meta-análise para examinar as relações entre personalidade e bem-estar subjetivo. Em se tratando do modelo dos Cinco Grandes Fatores, o Neuroticismo foi o mais forte preditor de satisfação com a vida, felicidade e afeto negativo. $\mathrm{O}$ afeto positivo, por sua vez, foi melhor predito por Extroversão e Socialização. No entanto, os autores ressaltam que para compreender plenamente o bem-estar subjetivo, deve-se também analisar fatores situacionais e variáveis demográficas, como por exemplo, os eventos diários, os recursos pessoais e a saúde. Para eles, há certo exagero na determinação da importância atribuída à extroversão para o bem-estar subjetivo. 
Uma década depois, Steel, Schmidt e Shultz (2008) também realizaram uma meta-análise e criticaram os achados de DeNever e Cooper (1998), uma vez que eles teriam subestimado os valores de correlação entre os construtos (em torno de $r=0,20$ ), ao não considerarem a semelhança conceitual importante entre personalidade e bem-estar subjetivo, bem como superestimaram o pequeno impacto dos fatores situacionais. $\mathrm{Na}$ análise mais recente, os resultados indicaram que escalas de personalidade e de bem-estar subjetivo podem ser substancialmente diferentes, embora a relação entre os dois conceitos seja muito maior (por exemplo, quatro vezes) do que o preconizado pelas as meta-análises anteriores.

Sumarizando, traços de personalidade são importantes preditores de bem-estar subjetivo. O escopo de pesquisas que associam afetos e personalidade não é pequeno, no entanto, no Brasil, ainda é incipiente. Nesse ensejo, a presente pesquisa teve como objetivo verificar a existência de associação entre fatores de personalidade e afetos positivos e negativos em uma amostra de estudantes do Ensino Médio, menos frequentemente representada nos estudos brasileiros.

A este respeito, parece ser necessária uma consideração, pois em que pese o fato de que estudantes universitários compõem mais comumente as amostras das investigações, aquelas com outros participantes devem também ser realizadas, a fim de elucidar se as considerações feitas com base em adultos podem ser generalizadas para adolescentes. Nesse particular, Diener et al. (2002) defendem que pesquisas devem ser continuadas com o construto, dentre as quais as que avaliem sua estabilidade, por meio de estudos com crianças, jovens e adultos, assim como as que afiram os mecanismos que operaram e suas relações com outros construtos.

\section{Método}

\section{Participantes}

A amostra foi composta por 529 estudantes do Ensino Médio de cinco escolas, três públicas e duas particulares, escolhidas por conveniência, localizadas em uma cidade do interior de São Paulo. Os alunos participaram da pesquisa de maneira voluntária. As idades dos participantes variaram de 14 a 27 anos, com média de 16 anos $(D P=1,48)$. No que se refere ao sexo, 223 $(42,2 \%)$ eram homens e 306 (57,8\%), mulheres. Em relação à classificação dos participantes por ano escolar, foi observada uma distribuição razoavelmente igualitária na amostra quanto à série, sendo que $187(35,3 \%)$ cursavam o primeiro ano, 164 (31\%) o segundo e $178(33,6 \%)$ o terceiro. Quanto ao sistema de ensino, 63,5\% ( $N=336)$ eram estudantes do sistema particular e $35,9 \%(N=190)$ estudantes do sistema público.

\section{Instrumentos}

Escala de Afetos Zanon - EAZ (Zanon, Bastianello, Pacico, \& Hutz, 2013b). Composta por 20 afirmações que descrevem sentimentos e emoções passadas e presentes, como por exemplo, "Sou apaixonado por algumas coisas que eu faço". O instrumento é autoaplicável, sendo necessária apenas a folha de resposta, a qual contém a descrição dos itens. O respondente assinala a resposta numa escala tipo Likert de 5 pontos, variando de 'nada a ver com você' (1 ponto) a 'tudo a ver com você' (5 pontos).

Sua construção partiu de um estudo inicial, no qual 853 universitários ( $57 \%$ mulheres), com média de idade de 21 anos ( $D P=3)$, responderam a 29 itens, constantes na primeira versão do instrumento, os quais foram submetidos à análise de componentes principais, por rotação Oblimin. Não foram encontrados índices satisfatórios de consistências interna para o modelo de três e quatro fatores, além de apresentarem diversos itens com cargas fatoriais acima de 0,35 em mais de um fator, o que não fazia sentido teoricamente. Portanto, não foi considerada pertinente essa distribuição. Os resultados da análise componencial mostraram que o índice Kaiser-Meyer-Olkin foi de 0,90 e o teste de esfericidade de Bartlett foi significativo $(p<0,001)$. A solução bifatorial produziu o primeiro fator com eigenvalue de 5,1 , que explicou $25,6 \%$ da variância total e o segundo fator com eigenvalue de 2,6, que explicou $12,8 \%$ da variância total. A consistência interna da escala, avaliada pelo alfa de Cronbach, foi de 0,83 para afeto positivo e 0,77 para afeto negativo. As evidências de validade foram buscadas por meio das correlações entre a EAZ e a Positive and Negative Affect Schedule (PANAS: Watson et al., 1988). Observaram-se índices altos de correlação entre os afetos positivos $(0,73)$ e afetos negativos $(0,74)$, o que indica que as subescalas da EAZ medem o mesmo construto das subescalas da PANAS.

O segundo instrumento, a Bateria Fatorial de Personalidade - BFP (Nunes, Hutz, \& Nunes, 2010), é composta por 126 itens que descrevem sentimentos, opiniões e atitudes. Os itens contemplam Neuroticismo, Socialização, Realização, Abertura para experiência e Extroversão. As respostas são registradas numa escala do tipo Likert de 7 pontos de acordo com o quanto o 
sujeito se identifica com cada sentença. Trata-se de um instrumento psicológico construído para avaliação da personalidade a partir do modelo dos cinco fatores (CGF). Esse instrumento compõe a lista dos testes aprovados para uso profissional no Brasil. Para o estudo normativo contou-se com uma amostra de 6599 pessoas, sendo a maioria estudantes universitários ou de ensino médio, provenientes de 11 estados brasileiros.

As análises fatoriais exploratórias indicaram a presença de cinco fatores coerentes com o modelo teórico, ficando compostos, o fator Neuroticismo por 29 itens $(\alpha=0,89)$; Extroversão, 25 itens $(\alpha=0,84)$; Socialização, 28 itens ( $a=0,85$ ); Realização, 21 itens ( $a=0,83)$; e Abertura, 23 itens $(a=0,74)$. Os eigenvalues variaram de 4,59 (Abertura) a 10,04 (Neuroticismo), com um total de variância explicada de $28,12 \%$. A partir dos itens que compuseram cada fator, foram identificadas para o fator Extroversão as facetas Comunicação (E1), Altivez (E2), Dinamismo (E3) e Interação Social (E4). Para o fator Socialização, as facetas Amabilidade (S1), Pró-sociabilidade (S2) e Confiança nas Pessoas (S3). Já para o fator Realização, foram definidas as facetas Competência (R1), Prudência/ Ponderação (R2) e Empenho/Comprometimento (R3). Fator Abertura as facetas Interesse por novas idéias (A1), Liberalismo (A2) e Busca por novidades (A3). Por fim, o fator Neuroticismo apresentou as facetas Vulnerabilidade (N1), Instabilidade Emocional (N2), Passividade/Falta de energia (N3) e Depressão (N4).

\section{Procedimentos}

A pesquisa foi realizada após encaminhamento do projeto de pesquisa para um Comitê de Ética. Mediante aprovação (Protocolo de Aprovação 24/2013), iniciou-se o procedimento de coletas de dados que durou cerca de três meses. Posteriormente ao contato com as escolas para autorização, foram contatados os estudantes, para que fossem informados dos objetivos da pesquisa e orientados quanto à assinatura do Termo de Consentimento Livre e Esclarecido (TCLE). A aplicação dos instrumentos foi feita coletivamente, em grupos de aproximadamente 30 estudantes, em dias e horários previamente estabelecidos. Os participantes responderam os dois instrumentos em uma única sessão, sendo primeiramente aplicada a BFP e em seguida a Escala de Afetos. Cada sessão durou em média 1 hora e 15 minutos.

\section{Procedimentos de análise de dados}

Para alcançar os objetivos do presente estudo, os dados dos instrumentos EAZ e BFP foram analisados por meio da correlação de Pearson. Além disso, foram realizadas correlações de Pearson entre EAZ e as facetas dos cinco fatores da BFP, quais sejam, Extroversão (Comunicação, Altivez, Dinamismo, Interação Social), Socialização (Amabilidade, Pró-Sociabilidade, Confiança nas pessoas), Realização (Competência, Ponderação, Empenho), Abertura (Abertura a ideias, Liberalismo, Busca por novidades) e Neuroticismo (Vulnerabilidade, Instabilidade, Passividade e Depressão).

\section{Resultados e discussão}

O presente estudo buscou investigar as associações entre os fatores de personalidade e os afetos positivos e negativos. A Tabela 1 informa os resultados das correlações e os respectivos níveis de significância.

Tabela 1. Correlações entre EAZ e os fatores da BFP

\begin{tabular}{lcccccc}
\hline EAZ & \multicolumn{7}{c}{ BFP } \\
\hline & & Neuroticismo & Extroversão & Socialização & Realização & Abertura \\
Positivos & $r$ & $-0,37^{* *}$ & $0,42^{* *}$ & $0,14^{*}$ & $0,34^{* *}$ & $0,09^{*}$ \\
Negativos & $r$ & $0,62^{* *}$ & 0,001 & $0,37^{* *}$ & 0,06 & $0,09^{*}$ \\
\hline${ }^{*} p<0,05 ;{ }^{* *} p<0,001$. & & & &
\end{tabular}

Das dez correlações entre EAZ e os cinco fatores da BFP, apenas duas não foram significativas, embora as magnitudes tenham variado de baixas a moderadas. $\mathrm{O}$ coeficiente mais forte foi encontrado entre Neuroticismo e afetos negativos, o que está em consonância com os achados de outras pesquisas (Albuquerque et al., 2012; Anglim \& Grant, 2014; Zanon et al., 2013b, dentre outros). Em relação aos afetos positivos, a maior correlação se deu com Extroversão, corroborando os dados de Albuquerque et al. (2012) e Steel, Schmidt e Shultz (2008). Ainda, o estudo de Brajša-Žganec et al. (2011) revelou que a estabilidade emocional foi o melhor preditor de ausência de afeto negativo e a extroversão foi o preditor de afeto positivo e satisfação com a vida. Analogamente, Diener, Suh e Oishi (1997) sugerem que os extrovertidos experimentam mais afetos positivos e que os neuróticos têm maiores níveis de afetos negativos. Para o melhor entendimento dos resultados foram realizadas correlações com as facetas da BFP. A Tabela 2 indica os resultados obtidos entre EAZ e as facetas de Extroversão.

Tabela 2. Correlações entre a EAZ e as Facetas do Fator Extroversão

\begin{tabular}{lccccc}
\hline EAZ & \multicolumn{5}{c}{ Extroversão } \\
Positivos & $r$ & $0,32^{* *}$ & $0,17^{*}$ & $0,48^{* *}$ & $0,31^{* *}$ \\
Negativos & $r$ & $-0,15^{*}$ & $0,26^{* *}$ & $-0,10^{*}$ & $-0,02$ \\
\hline${ }^{*} p<0,05 ;{ }^{* *}$ & $p<0,001$. & & &
\end{tabular}


Costa e Widiger (2002) apontam que Extroversão está associada à quantidade e à intensidade das interações interpessoais preferidas, nível de atividade, necessidade de estimulação e capacidade de alegrar-se. Altos índices de Extroversão podem revelar pessoas sociáveis, ativas, falantes, otimistas e afetuosas. Especificamente, a faceta dinamismo indica o quanto as pessoas são capazes de tomar iniciativas nas diversas situações, sendo que foi a que apresentou coeficiente de maior magnitude em relação aos afetos positivos. Pessoas com altos escores nessa escala tendem a ser mais dinâmicas, envolvem-se em várias atividades ao mesmo tempo e gostam de manter-se ocupadas mesmo nas situações de lazer. Embora, com coeficiente de menor magnitude que a faceta dinamismo, comunicação e interação social se relacionaram com afetos positivos com correlações em torno de $r=0,30$. Escores altos na faceta comunicação usualmente revelam facilidade para falar em público, falar mais sobre si e facilidade para conhecer pessoas novas. Além disso, as pessoas com altos escores tendem a iniciar conversas com outras pessoas, expressar suas opiniões e interesses quando estão em grupo e dificilmente sentem-se constrangidas em situações sociais (Nunes et al., 2010). Por sua vez, interação social descreve aqueles indivíduos que buscam situações que propiciem contato com outras pessoas. Assim, pode-se compreender a relação de ambas as facetas com afetos positivos, pois eles dizem respeito ao quanto uma pessoa está se sentindo entusiasmada, ativa e alerta (Snyder \& Lopes, 2009).

Com relação aos afetos negativos, a faceta altivez foi a que se correlacionou mais expressivamente, embora o coeficiente tenha sido baixo $(r=0,26)$. Essa faceta descreve pessoas com uma percepção grandiosa sobre a sua capacidade e valor. Indivíduos com escores altos nessa escala relatam a necessidade de receber atenção, têm a crença de que os outros podem invejá-la e predisposição para falar sobre si (Nunes et al., 2010), o que parece ser coerente com o conceito de afeto negativo, qual seja, diz respeito à angústia, insatisfação, raiva, culpa, desgosto e medo (Watson et al., 1988).

Os resultados obtidos em relação às facetas de Extroversão, não estão em concordância com os dados de Nunes et. al. (2009), uma vez que os autores encontraram a maior relação com interação social, não com dinamismo, como no presente estudo. A este respeito, pode-se inferir que as diferentes amostras entre o estudo dos autores e o presente, explique, se não totalmente, mas parcialmente, os dados. Possivelmente a interação social entre adolescentes não esteja tão estabilizada quanto ocorre com adultos. No entanto, em relação à correlação entre Extroversão e afetos positivos, há consonância com os resultados de Brajša-Žganec et al. (2011) e Anglim e Grant (2014). A seguir, na Tabela 3 encontram-se os resultados das correlações entre EAZ e o fator Socialização.

Tabela 3. Correlações entre a EAZ e as Facetas do Fator Socialização

\begin{tabular}{lcccc}
\hline EAZ & \multicolumn{3}{c}{ Socialização } \\
\hline & & Amabilidade & Pró-Sociabilidade & Confiança nas Pessoas \\
Positivos & $r$ & $0,15^{*}$ & 0,04 & 0,13 \\
Negativos & $r$ & $-0,07$ & $-0,30^{* *}$ & $-0,47^{* *}$ \\
\hline
\end{tabular}

Socialização descreve a qualidade das relações interpessoais dos indivíduos podendo representar pessoas que são generosas, bondosas, afáveis, prestativas, altruístas, interessadas e disponíveis para ajudar os outros; tendem a ser responsivas e empáticas, e acreditam que os outros agirão da mesma forma (Nunes et al., 2010). No presente estudo, os afetos negativos se correlacionaram moderada e negativamente com pró-sociabilidade e confiança nas pessoas. A última em especial, revela o quanto as pessoas acreditam em outras, de modo que escores muito baixos revelam uma forte percepção de que as pessoas podem prejudicá-las. Assim, pode-se compreender, em relação aos resultados presentes, que as pessoas que não acreditam nas outras e têm dificuldade de se vincular, possuem mais afetos negativos. Merece também destaque a correlação entre pró-sociabilidade e afetos negativos $(r=-0,30)$. As pessoas que têm baixos resultados na faceta podem ser manipuladoras, de modo que os outros acabam fazendo o que elas desejam. Costumam ser hostis na interação com os demais, tratando-os de forma desrespeitosa ou opositora (Nunes et al., 2010). Assim, é presumível que possuam mais afetos negativos, à medida que eles revelam uma dimensão geral da angústia e insatisfação, e incluem estados de humor aversivos (Diener et al., 2002). A seguir a Tabela 4 dispõe os resultados referentes às facetas do fator Realização.

Tabela 4. Correlações entre a EAZ e as Facetas do Fator Realização

\begin{tabular}{lcccc}
\hline EAZ & \multicolumn{3}{c}{ Realização } \\
\hline & & Competência & Ponderação & Empenho \\
Positivos & $r$ & $0,52^{* *}$ & 0,06 & $0,27^{* *}$ \\
Negativos & $r$ & $-0,07$ & $-0,12^{*}$ & 0,06 \\
\hline${ }^{*} p<0,05 ;{ }^{* *} p<0,001$. & & &
\end{tabular}


A. P. P. Noronha, D. F. Martins, R. R. F. Campos, C. S. M. Mansão

O fator Realização descreve características como o grau de organização, persistência, controle e motivação, sendo que pessoas com escores altos em realização tendem a ser organizadas, confiáveis, trabalhadoras, decididas, pontuais, ambiciosas e perseverantes. Pessoas com baixos escores em realização não têm objetivos claros, tendem a ter pouco comprometimento e responsabilidade diante de tarefas e geralmente são descritas como sendo preguiçosas, descuidadas, negligentes e hedonistas (Costa \& Widiger, 2002).

Os afetos positivos se associaram moderadamente com competência, que descreve uma atitude ativa na busca dos objetivos. Pessoas com afetos positivos tendem a ter uma percepção favorável de si mesmas, acreditando na sua capacidade para realizar as ações consideradas difíceis e importantes, o que pode justificar a associação entre afetos positivos e essa faceta. Os achados corroboram, por exemplo, os resultados de Albuquerque et al. (2012), cuja correlação mais forte também se deu com competência, embora com coeficiente menor ( $r=0,35)$ e de Brajša-Žganec et al.(2011). A Tabela 5 apresenta os achados referentes às facetas do fator abertura.

Tabela 5. Correlações entre a EAZ e as Facetas do Fator Abertura

\begin{tabular}{lcccc}
\hline EAZ & \multicolumn{3}{c}{ Abertura } \\
\hline & & Abertura a ideias & Liberalismo & Busca por novidades \\
Positivos & $r$ & 0,09 & 0,03 & 0,07 \\
Negativos & $r$ & $-0,11$ & $0,21^{* *}$ & 0,08 \\
\hline
\end{tabular}

${ }^{* *} p<0,001$.

As facetas de Abertura, assim como as de Realização, apresentaram menos coeficientes significativos com afetos. Houve uma única associação altamente significativa, de magnitude baixa, entre afetos negativos e liberalismo, que descreve uma tendência à abertura para novos valores morais e sociais. As pessoas que se identificam com esses itens tendem a relativizar valores morais, tendo consciência de que eles evoluem ao longo do tempo e que podem ser diferentes a depender da cultura local em questão. Os itens indicam a consciência de que os aspectos tidos como "verdades" tendem a mudar ao longo do tempo, do mesmo modo que as regras e costumes sociais (Nunes et al., 2010). É possível hipotetizar que as pessoas que vivenciam mais afetos negativos, e em razão disso, se sentem mais tristes, magoadas ou culpadas tendam a atribuir menor importância às "verdades absolutas", sendo assim mais opositoras. $\mathrm{Na}$ Tabela 6 estão dispostos os resultados referentes às facetas do fator Neuroticismo.
Tabela 6. Correlações entre a EAZ e as Facetas do Fator Neuroticismo

\begin{tabular}{lccccc}
\hline EAZ & \multicolumn{5}{c}{ Neuroticismo } \\
Positivos & $r$ & $-0,29^{* *}$ & $-0,13^{*}$ & $-0,33^{* *}$ & $-0,40^{* *}$ \\
Negativos & $r$ & $0,41^{* *}$ & $0,61^{* *}$ & $0,32^{* *}$ & $0,50^{* *}$ \\
\hline${ }^{*} p<0,05 ;{ }^{* *} p<0,001$. & & &
\end{tabular}

Neuroticismo refere-se ao nível crônico de ajustamento e instabilidade emocional dos indivíduos e padrões emocionais associados a desconforto psicológico (aflição, angústia, sofrimento) e os estilos cognitivos e comportamentais decorrentes. Pessoas com escores altos em Neuroticismo são propensas a vivenciar mais intensamente sofrimento emocional, são ansiosos, hostis, mais deprimidas, possuem baixa autoestima e tendem a ser impulsivas (Costa \& Widiger, 2002).

Todas as facetas de Neuroticismo associaram-se significativamente com afetos negativos, sendo que com instabilidade o coeficiente foi de magnitude alta. De modo geral, a interpretação negativa feita por pessoas com altos níveis de neuroticismo sobre os eventos de vida tende a estar associada a baixo afeto positivo e afetos negativos mais altos. Tanto o Neuroticismo, quanto os afetos negativos podem ser definidos em termos de estados de humor aversivos, o que reafirma a aproximação entre os construtos. Os achados são concordantes com os de Nunes et al. (2009), Albuquerque et al. (2012) e Anglim e Grant (2014).

Há que se destacar dois coeficientes significativos entre afetos positivos e as facetas depressão e vulnerabilidade, ambos negativos. A depressão, sob a perspectiva do BFP, indica baixa expectativa em relação ao futuro, sentimento de solidão e dificuldade para lidar com as adversidades, enquanto vulnerabilidade é uma faceta composta por indicadores de personalidade dependente e de esquiva, medo de receber críticas e insegurança (Costa \& McCrae, 1980). Desse modo, supõe-se que quanto mais depressão e vulnerabilidade são vivenciadas, menos afetos positivos estão presentes na vida destas pessoas, o que justificaria o resultado.

\section{Considerações finais}

Tomando como base as asserções de Zanon e Hutz (2010) ao enfatizarem as inter-relações existentes entre os construtos traços de personalidade e afetos, o presente estudo pretendeu investigar tais associações por meio de dois instrumentos de medida em uma amostra menos frequentemente estudada a este respeito no cenário nacional. Pesquisas dessa natureza permitem, 
mais que aplicar e avaliar resultados, confirmar ou rejeitar modelos teóricos, bem como tecer particularidades para amostras específicas quanto ao funcionamento e desenvolvimento de características psicológicas. Evidências vêm sendo apresentadas na literatura de que a influência da personalidade no bem-estar pode ser significativa (Chan \& Joseph, 2000; Garcia \& Erlandsson, 2011; Lucas \& Diener, 2008), o que permite afirmar que fatores de personalidade constituem-se como fortes preditores dos afetos positivos e negativos, componentes do bem-estar subjetivo. As análises realizadas no presente estudo revelam resultados que corroboram essas evidências, uma vez que foram encontradas importantes associações entre os fatores dos Cinco Grandes Fatores de Personalidade e Afetos Positivos e Negativos.

Os achados apontam correlações fortes entre neuroticismo e afetos negativos. As quatro facetas desse fator apresentaram associações significativas com afetos negativos, embora a faceta instabilidade tenha se destacado com coeficiente de alta magnitude. No que se refere aos afetos positivos, a maior correlação se deu com Extroversão, recorrentemente citada na literatura como a mais forte preditora. Em particular, a faceta dinamismo foi a que apresentou coeficiente com maior magnitude.

Outros estudos têm utilizado o modelo dos Cinco Grandes Fatores na tentativa de confirmar a associação entre personalidade e bem-estar, e os resultados têm indicado que principalmente Extroversão e Neuroticismo associam-se aos afetos (Albuquerque et al., 2012; Barros, 2013; Anglim \& Grant, 2014; dentre outros). Sendo assim, tanto as correlações apresentadas no presente estudo como de outros autores parecem indicar que a felicidade está associada a altos níveis de extroversão e baixos de neuroticismo.

Importante se faz destacar que os achados dessa investigação referem-se a uma amostra de estudantes do Ensino Médio o que confere originalidade ao estudo uma vez que a maior parte dos estudos sobre a temática em questão têm sido desenvolvidos com adultos. No entanto, os achados não se diferenciaram tão completamente de outros, cujas amostras foram compostas por adultos. Algumas diferenças referem-se às facetas de Neuroticismo, depressão e vulnerabilidade, que apresentaram correlações negativas com afetos positivos. Possivelmente os resultados estão de acordo com características desenvolvimentais dos adolescentes do estudo, uma vez que os jovens têm incertezas em relação ao futuro, inseguranças e medo de críticas. A faceta liberalismo também permite uma reflexão interessante. Ela correlacionou-se positivamente com afetos negativos, o que permite inferir que os valores morais, fortemente questionados na adolescência, podem suscitar mais estados de humor aversivos. Assim, justifica-se a necessidade de investigações para uma compreensão mais ampliada dos afetos positivos e negativos e suas interações com personalidade visto que podem ocasionar um maior ou menor bem-estar subjetivo em amostras distintas.

Por fim, há que salientar as limitações do estudo, como o tamanho da amostra e sua localização, considerando que foram avaliados apenas estudantes de uma cidade do interior de São Paulo. Sugere-se, a título de agenda de pesquisas, que os estudos incluam participantes de outras regiões do país, bem como outros temas referentes a afetos e personalidade. Outra possibilidade relevante a fim de debater o dilema apresentado pelas meta-análises de Steel, Schmidt e Shultz (2008) e de DeNever e Cooper (1998), é que as variáveis pessoais e contextuais possam ser investigadas em estudos futuros, e que não estiveram incluídos no escopo do presente artigo.

\section{Referências}

Albuquerque, I., Lima, M. P., Matos, M., \& Figueiredo, C. (2012). Personality and subjective well-being: What hides behind global analyses? Social Indicators Research, 105, 447-460. doi: 10.1007/ s11205-010-9780-7

Albuquerque, I., Lima, M. P., Matos, M., \& Figueiredo, C. (2013). The interplay among levels of personality: The mediator effect of personal projects between the Big Five and subjective well-being. Journal of Happiness Studies, 14(1), 235-250. doi: 10.1007/s10902-012-9326-6

Alcalá, V., Camacho, M., Giner, D., Giner, J., \& Ibañez, E. (2006). Afectos y gênero. Psicothema, 18(1), 143-148. Recuperado de http://www. psicothema.com/psicothema. asp? $\mathrm{id}=3189$

Anglim, J., \& Grant, S. (2014). Predicting psychological and subjective well-being from personality: incremental prediction from 30 facets over the big 5. Journal of Happiness Studies, 15(5). doi: 10.1007/ s10902-014-9583-7

Argyle M. (1999). Causes and correlates of happiness. In: D. Kahneman, E. Diener, \& N. Schwarz (Eds). Well-being: The foundations of hedonic psychology (pp. 353-373). New York: Russell Sage Foundation.

Barros, M. V. C (2013). Afetos e suas relac@ões com interesses profissionais e personalidade: Alunos do ensino médio e universitários (Dissertação de Mestrado). Universidade São Francisco, Itatiba, São Paulo

Brajša-Žganec, A., Ivanovic', D., \& Lipovç̧an, L. K. (2011). Personality traits and social desirability as predictors of subjective well-being. Psihologijske Teme, 20(2), 261-276. Recuperado de http://pt.ffri.hr/ index.php/pt/article/view/2 
Brajša-Žganec, A., \& Kaliterna-Lipovčan, L. (2006). Quality of Life, Life Satisfaction and Happiness in Professional Care Givers. Journal for General Social Issues, 4(5), 713-728.

Chan, R., \& Joseph, S. (2000). Dimensions of personality, domains of aspiration, and subjective well-being. Personality and Individual Differences, 28, 347-354. doi:10.1016/S0191-8869(99)00103-8

Costa, P. T. Jr., \& McCrae, R. R. (1980). Influence of extraversion and neuroticism on subjective well-being. Journal of Personality and Social Psychology, 38, 668-678. doi.org/10.1037/0022-3514.38.4.668

Costa, P. T. Jr., \& Widiger, T. A. (2002). Introduction. In P. T. Costa \& T. A.Widiger (Orgs.), Personality disorders and the five-factor model of personality (pp. 3-16). Washington, D.C: American Psychology Association.

DeNever, K. M., \& Cooper, H. (1998). The happy personality: A meta-analysis of 137 personality traits and subjective well-being. Psychological Bulletin, 124(2), 197-229. doi: 10.1037/0033-2909.124.2.197

Diener, E., Lucas, R. E., \& Oishi, S. (2002). Subjective well-being: The science of happiness and life satisfaction. In C. R. Snyder \& S. J. Lopez (Orgs.), Handbook of positive psychology (pp. 63-73). Nova Iorque: Oxford University Press.

Diener, E.; Suh, E. M., \& Oishi, S. (1997). Recent findings on subjective well-being. Indian Journal of Clinical Psychology, 24, 25-41.

Garcia, D., \& Erlandsson, A. (2011). The relationship between personality and subjective well-being: Different association patterns when measuring the affective component in frequency and intensity. Journal of Happiness Studies, 12(6), 1023-1034. doi: 10.1007/ s10902-010-9242-6

Inglehat, R., \& Klingemann, H. D. (2000). Genes, culture, Democracy and Happiness. In E. Diener \& E. Suh, (Eds.) Subjective well-being across cultures (pp. 165-183). Cambridge: MIT Press.

Lucas, R. E., \& Diener, E. (2008). Personality and subjective well-being. In O. P. John, R. W. Robins \& L. A. Pervin (Orgs.), Handbook of personality theory and research (pp. 95-814). Nova lorque: Guilford.

Mroczek, D. K., \& Kolarz, C. M. (1998). The effect of age on positive and negative affect: A developmental perspective on happiness. Journal of Personality and Social Psychology, 75(5), 1333-1349.

Nemanick Jr., R. C., \& Munz, D. C. (1997). Extraversion and neuroticism, trait mood, and state affect: A hierarchical relationship. Journal of Social Behavior \& Personality, 12, 1079-1092.

Nunes, C. H. S., Hutz, C. S., \& Giacomoni, C. H. (2009). Associação entre bem estar subjetivo e personalidade no modelo dos cinco grandes fatores. Avaliação Psicológica, 8(1), 99-108. Recuperado de http://pepsic.bvsalud.org/scielo.php?script=sci_arttext\&pi$\mathrm{d}=$ S1677-04712009000100009\&lng=en\&tIng=pt
Nunes, C. H. S. S., \& Hutz, C. (2002). O modelo dos cinco grande fatores de personalidade. In R. Primi. Temas de Avaliação Psicológica. Campinas: IBAP.

Nunes, C. H. S., Hutz, C. S., \& Nunes, M. O. (2010). Bateria fatorial de personalidade - manual técnico. Casa do Psicólogo: São Paulo.

Segabinazi, J. D., Zortea, M., Zanon, C., Bandeira, D. R., Giacomoni, C. H., \& Hutz, C. S. (2012). Escala de afetos positivos e negativos para adolescentes: Adaptação, normatização e evidências de validade. Avaliação Psicológica, 11(1), 1-12. Recuperado de http://pepsic.bvsalud.org/scielo.php?script=sci_arttext\&pid $=$ S1677-04712012000100002

Seligman, M. E. P. (2004). Felicidade autêntica: usando a nova Psicologia Positiva para a realização permanente. Rio de Janeiro, RJ: Objetiva.

Seligman, M. E. P. (2011). Flourish. Nova Iorque, NY: Free Press.

Snyder, C. R., \& Lopez, S. S. (2009). Psicologia positiva: uma abordagem científica e prática das qualidades humanas. Porto Alegre: Artmed.

Steel, P., Schmidt, J., \& Shultz, J. (2008). Refining the relationship between personality and subjective well-being. Psychological Bulletin, 134(1), 138-161. doi: 10.1037/0033-2909.134.1.138

Warner, R. M., \& Rasco, D. (2014). Structural equation models for prediction of subjective well-being: Modeling negative affect as a separate outcome. The Journal of Happiness \& Well-Being, 2(1), 34-50. Recuperado de http://www.journalofhappiness.net/articles/ pdf/v02i01/MODEL.pdf

Watson, D., Clark, L. A., \& Tellegen, A. (1988). Development and validation of brief measures of positive and negative affect:The PANAS Scales. Journal of Personality and Social Psychology, 54, 1063-1070. doi: 10.1037/0022-3514.54.6.1063

Wilson, W. (1967). Correlates of avowed happiness. Psychological Bulletin, 67, 294-306. doi: 10.1037/h0024431

Zanon, C., \& Hutz, C. S. (2010). Estilos de pensamento, personalidade e bem-estar subjetivo: Avanços e polêmicas. Aletheia, 32(1), 80-91. Recuperado de http://pepsic.bvsalud.org/scielo.php?script=sci_artt ext\&pid=S1413-03942010000200007

Zanon, C., Bastianello, M. R., Pacico, J. C., \& Hutz, C. S. (2013a). Relationships between positive and negative affect and the five factors of personality in a Brazilian sample. Paidéia, 23(3), 285-292. doi: 10.1590/1982-43272356201302

Zanon, C., Bastianello, M. R., Pacico, J. C., \& Hutz, C. S. (2013b). Desenvolvimento e validação de uma escala de afetos positivos e negativos. PsicoUSF, 18(2), 193-201. doi: 10.1590/S141382712013000200003 
Relações entre afetos positivos e negativos e os cinco fatores de personalidade

Ana Paula Porto Noronha, Doutora em Psicologia Ciência e Profissão pela Pontifícia Universidade de Campinas (PUCCamp), é Docente do Programa de Pós-Graduação Stricto Sensu em Psicologia da Universidade São Francisco (USF). Endereço para correspondência: Rua Alexandre Rodrigues Barbosa, 45, Centro,

Itatiba, SP, CEP 13251-900. E-mail: ana.noronha@usf.edu.br

Denise da Fonseca Martins, é Mestre em Psicologia, com ênfase em Avaliação Psicológica, pela Universidade São Francisco (USF), e é Doutoranda em Psicologia, com ênfase em Avaliação Psicológica, pela Universidade São Francisco (USF). E-mail: denisefmart@gmail.com

Roberta Ramazotti Ferraz Campos, Mestre em Psicologia, com ênfase em Avaliação Psicológica, pela Universidade São Francisco (USF), é Doutoranda em Psicologia, com ênfase em Avaliação Psicológica, pela Universidade São Francisco. E-mail: roberta.rfcampos@gmail.com

Camélia Santina Murgo Mansão, Doutora em Psicologia Ciência e Profissão pela Pontifícia Universidade de Campinas (PUCCamp). Pós-doutorado em Avaliação Psicológica pela Universidade São Francisco

(USF), é Coordenadora do Programa de Mestrado em Educação da Universidade do Oeste Paulista (UNOESTE). E-mail: camelia@unoeste.br 